\title{
Functional Endoscopic Sinus Surgery in Children
}

V.P. Venkatachalam, Senior ENT Consultant, Department of Otorhinolaryngology Safdarjang Hospital, New Delhi - 110029

\section{Abstract}

Functional Endoscopic Sinus Surgery Which has been introduced in Europe by Messerklinger and Stammberger and later on in United States by Kennedy, has now become a standard modality of treatment for sinus diseases in our country. However, most of the work reported from our country pertain to adult population. In this paper, we present our experience in 30 children who underwent Functional Endoscopic Sinus Surgery over the past four years.

The age of the patients varied from 7-14 years. All the patients tolerated the procedure well and there was no major complication. Follow-up period varied from 9-39 months with a mean follow-up of 18.3 months. Out of 30 patients, 27 patients were available for long term assessment of results. 18 patients $(66.67 \%)$ reported complete improvement of symptoms, while 6 patients $(22.22 \%)$ had partial improvement. 3 patients $(11.11 \%)$ showed no improvement. The results of this small series reveal that Functional Endoscopic Sinus Suregery has a definite role in the treatment of sinus disease in children.

\section{Key Words :}

Functional Endoscopic Sinus Surgery, Children. 
T he concept of treatment of sinus disorders has changed with the development of Hopkin's rod system of endoscopes coupled with the introduction of Functional Endoscopic Sinus Surgery technique by Messerklinger ${ }^{(1)}$ and Stammerberger ${ }^{(2)}$ in Europe and by Kennedy ${ }^{(4)}$ in United States. Functional Endoscopic Sinus Surgery provides an important addition to our ability to surgically treat patients in whom medical treatment for sinus diseases fail. Although there is evidence that sinusitis in children can be cured with appropriate antibiotics yet a selective few are

Table - 1

Symptoms and Findings $(n=30)$

\begin{tabular}{|llr|}
\hline Symptoms & $::$ & \\
- Nasal Obstruction/congestion & $::$ & 30 \\
- Nasal Discharge & $::$ & 21 \\
- Hequency of URI & $::$ & 24 \\
- Nocturnal cough & $::$ & 6 \\
\hline Findings & $::$ & 12 \\
- Congestion & & \\
- Purulent Discharge & $::$ & 21 \\
- Polyps & $::$ & 6 \\
- Mucosal oedema & $::$ & 12 \\
- Septal Deviation & $::$ & 12 \\
\hline
\end{tabular}

refractory to medical treatment and will require surgical intervention.

The results with surgical procedures like tonsillectomy, adenotonisillectomy and nasoantral windows have been dismal in treating sinusitis in children. ${ }^{(5)}$

In this paper, we have tried to evaluate the role of functional endoscopic sinus surgery in 30 children suffering from sinus disorders, refractory to medical therapy, over a period of 4 years.

Table - 2

CT Findings $(n=30)$

\begin{tabular}{|lrr|}
\hline Pansinusitis & $::$ & 3 \\
Maxillary sinusitis & $::$ & 18 \\
Ethmoid & $::$ & 9 \\
Concha Bullosa & $::$ & 3 \\
Paradoxical Middle Turbinate & $::$ & 6 \\
\hline
\end{tabular}

Table - 3

Surgical Procedures Performed (n=39)

\begin{tabular}{llr|}
\hline Anterior Ethmoidectomy & $:$ & 15 \\
Total Ethmoidectomy & $::$ & 9 \\
Total Ethmoidectomy + & $::$ & 3 \\
Sphenoidotomy & $::$ & 12 \\
Polypectomy + Infundibulotomy & $:$ & \\
\hline
\end{tabular}

Concurrent Adenotonsillectomy, adenoidectomy was performed in one case each and in three patients-inferior turbinoplasty was performed.

\section{Material and Methods \\ *}

A total of 30 patients belonging to paediatric age group who were refractory to medical therapy were included in the study. After a detailed history followed by a thorough clinical examination, all the patients were subjected to a standard protocol of haematological and radiological examination (X-ray PNS Lateral view). A diagnostic nasal endoscopy was performed in majority of the cases under local anaesthesia and sedation. CT evaluation of the nose and paranasal sinuses was carried in all the cases. All the patients were put on 2 weeks course of antibiotic (amoxicillin-Clavilunate potassium) before being taken up for surgery. Along with endoscopic sinus surgery adenoidectomy and adenotonsillectomy was performed in one patient each and in three patients inferior turbinoplasty was performed to facilitate drainage and improve the nasal airway.

\section{Technique}

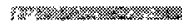

In 25 patients the procedure was performed under local anaesthesia and in 5 patients it was performed under general anaesthesia. Prior to operation the

Table -4

Surgical findings.

\begin{tabular}{|lcc|}
\hline Mucosol oedema & $::$ & 24 \\
Polyps & $::$ & 12 \\
Polypoid Mucosa & $::$ & 9 \\
Choanal Polyp & $::$ & 3 \\
Pus & $::$ & 6 \\
Concha Bullosa & $::$ & 3 \\
\hline
\end{tabular}


patients were under antibiotics and nasal decongestant spray. Those who underwent the procedure under local anaesthesia were premeicated with Injection Pethidine hydrochloride and Injection Promethazine hydrochloride as per their body weight. The nasal cavity was anaesthetized by $4 \%$ lignocaine by Moffet's technique first and later on packed with $4 \%$ lignocaine with adrenaline in $1: 100,000$ concentration for 10 minutes. After anaesthesia (both in cases of general and local), the area is infiltrated locally with a local anaesthetic agent (2\% lignocine with adrenaline in $1: 1000,000$ concentration) using a 26 gauge needle. The nose was then packed with $4 \%$ lignocaine with adrenaline (1:50,000 concentration) for 3 minutes. The standard Messerklinger technique FESS was employed. All the procedures were performed using $0^{\circ}$ or $30^{\circ} 4 \mathrm{~mm}$ telescope. The ethmoidal air cell were exenterated, frontal recess opened, any polyp present was removed and the maxillary ostia was enlarged to a size of 3-4 $\mathrm{mm}$. In three cases sphenoidotomy was performed. During the surgical procedure, squeezed cotton wool soaked in high concentration of adrenaline $(1: 30,000)$ was used to achieve haemostasis. At the end of operation Inj. Depomedrol/Inj. Dexamethasone was injected into cavity and packed with antibiotic steroid ointment smeared merocel pack. The pack, besides preventing adhesion between middle turbinate and lateral wall, also helps in controlling any postoperative bleeding. Postoperatively Merocel Pack was removed 72 hours after surgery. The patients were put on a saline nasal douche, nasal decongestant drop, steroid spray and Mucolytic agent, and antibiotics for 2 weeks. Two week after the surgery a nasal endoscopy was performed to clear the cavity of the clots granulation tissue and remove any synchiae. At the end of it the cavity was again packed with steroid antibiotic cream. The patients were followed at weekly intervals initially, later on at fortnightly and monthly intervals.

\section{Observations and Results}

The age of the patients varied from 7-14 years with a mean age of 10.5 years $(7-8, n=3 ; 9-10, n=9 ; 11-12$, $n=12 ; 13-14, n=6)$. Males outnumbered females by $2: 1$ ratio. Table-1 shows the presenting symptoms and clinical findings. X-ray paranasal sinuses revealed hazziness of maxillary sinus in 21 cases, frontal sinus in 3 cases and ethmoid sinus in 12 cases. Besides these findings, the maxillary sinus showed thickening of the mucosa in 12 cases, rounded shadow/polyp in 9 cases and fluid level in one case. A diagnostic nasal endoscopy was performed in all the cases and it revealed mucopus and obstructed ostiomeatal complex in all the cases. The other findings included polyp in 12 cases, paradoxical middle turbinate in 4 cases and deviated nasal septum and concha bullosa in 3 cases each. A coronal CT of nose and paranasal sinuses was carried out in all the cases and the findings are enumerated in Table-2. Patients were subjected to endoscopic sinus surgery and the various operations performed as well as the findings are shown in Table3 and 4 . The follow-up period varied from 9 months - 39 months with a mean follow-up of 18,3 months. The results were evaluated on the basis of improvement of symptoms. Both the patients as well as parents were asked about the improvement. Out of 30 patients who underwent surgery, 27 patients could be evaluated and 3 patients were lost to follow-up 18 patients $(66.67 \%)$ had complete relief of symptoms, while 6 patients $(22.22 \%)$ had partial relief of symptoms. 3 patients $(11.22 \%)$ showed no improvement. There was no major operative complication. However, 2 patients had bleeding due to injury to anterior ethmoidal artery which was controlled by anterior nasal packing. 3 patients had synechea and 6 others had meatal closure.

\section{Discussion \\ sux}

The aim of Functional Endoscopic Sinus Surgery is to establish normal drainage and ventilation of the paranasal sinuses by removing diseased mucosa and opening the obstructed ostiomeatal complex, the area of the middle meatus where the frontal, maxillary and ethmoid sinuses mucoliliary flow converge. Apart from anatomical changes, the commonest causes for the obstruction at the region are upper respiratory tract infection and allergy. It has been observed that ant rostomies do not address to ostiomeatal complex or the other sinuse- in children. The diagnosis of sinusitis in children is difficult and is usually based on clinical 
manifestations which include cough, rhinorrhoea and repeated URI. Radiographic evaluation of the sinuses is of paramount importance in diagnosis as well as treatment in children. We use Functional Endoscopic Sinus Surgery in children only, when the patient fails to respond to conservative treatment i.e. two weeks treatment of antibiotics, steroid spray and nasal decongestants.

In children in spite of the anatomical variation the author has been able to successfully employ $43 \mathrm{~mm}$ telescope for surgical procedure. Prior to surgery it is mandatory for the surgeon to assess the status of adenoids, tonsils and inferior turbinate since hypertrophy of these structures may adversely affect the sinonasal physiology and contribute to recurrent or chronic infection of the sinuses.

Excellent visualisation during the procedure is must for the success of the surgical technique and for this it is mandatory to wait strictly for 10 minutes after applying vasoconstrictor packs in the nose. Postoperative management include putting the patient on antibiotics, nasal decongestants, saline douch and steroid spray. A nasal endoscopy is performed after 2 weeks of surgery under sedation and local anaesthesia to remove any adhesions and synechiae from the cavity.

The results of this series compare favourably with our earlier series in adult patients ${ }^{6}$ as well as with that of other authors who have reported their results of paediatric endoscopic sinus surgery. ${ }^{7,89}$ The safety of this procedure is demonstrated by lack of any major complication in this series. This series demonstrated that Functional Endoscopic Sinus Surgery in children is safe provided one follows the strict principle of proper patient selection as well as haemostasis during the procedure. The effectiveness of this modality of treatment is well demonstrated by improvement of symptoms.

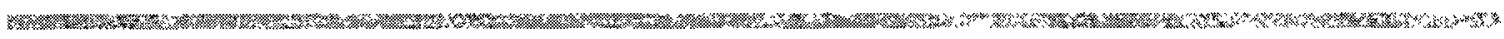

\section{References \\ r.}

1. Messerklinger W (1978) : Endoscopy of the nose. Urban and Schwarzenberg (eds), Baltimor, Munich.

2. Stammberger H (1986) : Endoscopic Endonasal surgery - Concepts in treatment of Recurring Sinusitis. Part l. Anatomic and Pathophysiological consideration. Otolaryngol Head and Neck Surg 94 (2) : 143 - 146.

3. Stammberger (1986) : Endoscopic Endonasal Surgery - Concepts in treatment of Recurring Sinusitis Part II Results. Otolaryngol Head and Neck Sug. 92(2) : 147 - 156.

4. Kennedy, DN, Zinreich, S.J., Rosen Baum AE, et al (1985) : F Functional Endoscopic Sinus Surgery. Theory and Diagnostic Evaluation. Arch Otolaryngol 111 : 576 - 582.

5. Munkz HR and Lusk RP (1990) : Nasal Antral Windows in children : A Retrospective study. Laryngoscope $100: 643-646$.

6. V.P. Venkatachalam and Arun Bhat (1997) : Functional Endoscopic Sinus Surgery. Initial Results Part II, J.K. Practitioner Vol. 4 No. 4, 277-279.

7. Gross CN, Guruchari MS, Lazar R and Long TE (1989) : Functional Endoscopic Sinus Surgery in the Paediatric Age Group. Laryngoscope 99 : 272-275.

8. Stankiewicz JA (1995) : Paediatric Endoscopic Nasal and Sinus Surgery. Otolaryngol Head and Neck Surgery 113(3) : 204-210.

9. Munkz RP and Lusk HR (1990) : Endoscopic sinus surgery in children with chronic sinusitis: A Pilot Study Laryngoscope $100: 654-658$. 